\title{
Structure of neighborhoods in a large social network
}

\author{
Alina Stoica \\ Orange Labs and Liafa \\ Paris, France \\ stoica@liafa.jussieu.fr
}

\author{
Christophe Prieur \\ Liafa \\ Paris, France \\ prieur@liafa.jussieu.fr
}

\begin{abstract}
We present here a method for analyzing the neighborhoods of all the vertices in a large graph. We first give an algorithm for characterizing a simple undirected graph that relies on enumeration of small induced subgraphs. We make a step further in this direction by identifying not only subgraphs but also the positions occupied by the different vertices of the graph. We are thus able to compute the roles played by the vertices of the graph, roles found according to a new definition that we introduce. We apply this method to the neighborhood of each vertex in a $2.7 M$ vertices, $6 M$ edges mobile phone graph. We analyze how the contacts of each person are connected to each other and the positions they occupy in the neighborhood network. Then we compare their quantity of communication (duration and frequency) to their positions, finding that the two are not independent. We finally interpret and explain the results using social studies on phone communications. ${ }^{1}$
\end{abstract}

\section{Categories and Subject Descriptors}

H.2.8 [Database Management]: Database ApplicationsData Mining; G.2.2 [Discrete Mathematics]: Graph Theory-Graph algorithms; J.4. [Computer Applications]: Social and Behavioral Sciences-Sociology

\section{General Terms}

Algorithms, Human Factors, Measurement, Theory

\section{Keywords}

social networks, roles, patterns, complex networks, personal networks

\section{INTRODUCTION}

The study of social networks has changed a lot since the early pioneering works of anthropologists who decided to focus

\footnotetext{
${ }^{1}$ Supplementary material is available online at www.liafa.jussieu.fr/ ${ }^{\sim}$ stoica/neighborhoods
}

on relationships instead of individuals $[7,4,3]$. After the technical framework of social network analysis was settled in the 1970's by the combination of mathematical tools such as graph theory, algebra and statistics [26, 8, 32, 33], the field has been again shaken with the exponential growing of the size of relational databases coming with the development of communication tools. The tremendous research activity on the structure of the World-Wide Web [14, 11, 12] that have pre-dated Google's PageRank algorithm [9] have given birth to a new object of study, namely complex networks, due to the common properties found to be shared not only by the graph of the WWW $[2,34]$ but also by many networks appearing in various contexts (biology, linguistics, economics and, of course, social networks) $[29,6]$.

There is thus a wide gap between these kinds of studies of the global structure of huge networks and qualitative studies of personal networks, sometimes built from face-to-face interviews (for a historical survey of this trend, see [35]), even though more and more such studies now take as data personal networks scraped from internet's social network services [18]. Inbetween, the classical problem of identifying roles in a (possibly quite large) network, introduced in the 1970 's as one of the main tools of social network analysis [37], relies on the fact that some nodes have similar positions in the sense that they are linked to the same other nodes, which is defined as the so-called structural equivalence of nodes, or the more general notion of regular equivalence, where two nodes are equivalent if the neighbors of the two are equivalent to each other [5].

Our work is at the intersection of these three research trends: we study the roles of nodes in the personal networks of all individuals of a large (thus 'complex') network. In [31] (in French), we already compared to a classical ethnographic study what can be achieved in terms of qualitative analysis with such a large-scale (2 million) collection of personal networks.

\footnotetext{
Now to address the issue of roles, we devised a method relying on a very popular data mining problem: the search for frequent subgraphs in a given (possibly large) graph. On this issue, some authors considered that frequent subgraphs are the ones that appear in a given graph (or set of graphs) more often than a chosen threshold. Some algorithms $[19$, $21]$ extend the apriori-based candidate generation-and-test approach [1], while others [5, 39] use a pattern-growth approach [15]. More recently, several algorithms have been
} 
proposed for significant graph pattern mining [17, 38]. Milo et al [28] used another approach to find interesting patterns. They compared the frequency of subgraphs with the ones appearing in randomly generated graphs that share some properties of the network. Several methods for an efficient counting of subgraphs with a given maximal size have been proposed since $[36,20]$. Here, we apply the method introduced by Wernicke to the neighborhood of each vertex of the given network. This allows us to compute in the same time the subgraphs that appear more frequently than a chosen threshold and the ones that appear more frequently than in randomly generated networks. Unlike previously done, to our knowledge, we are also able to identify the positions that the different neighbors occupy and therefore the roles they play.

We apply this method to a large network built from mobile phone communications. Of course, there are many forms of social interactions between two people: face-to-face interactions, emails, instant messages, (fixed) telephone, the mobile phone communications capturing only a subset of the underlying social network. However, studies on the strength of ties have shown that mobile phone is among the most intimate communication tools and moreover that people a mobile phone conversation suggests a certain relation between the two individuals, given that there aren't any listings of mobile phone numbers. Moreover, people that contact each other via one communication tool tend to communicate via other ones as well [16], hence the relevance of analyzing a mobile phone network in the search of understanding the underlying social network.

Different properties have been already identified in large mobile phone networks $[30,10]$. Onnela et al [30] show with no surprise that the distributions of degree and of the duration of calls are power-laws. More strikingly they also give a definition for the strength of ties depending on the duration of calls and they analyze the connection between the strength and the connectivity or the community structure.

As in complex network studies, all of these properties are global, characterizing the structure of the mobile phone graph as a whole. Here, our aim is to identify the local structure, the way the persons contacted by a given individual (ego) are connected to each other relatively to their "importance" to ego. In order to do that, we use the frequency and the total duration of communications between ego and each of his contacts.

The paper is organized as follows. After recalling some basic definition on graphs (Section 2), we detail in Section 3 a formal framework to address the issue of characterizing a graph in terms of a position equivalence along with an algorithm to do it. The main algorithm to characterize all the neighborhoods of a large graph is given in Section 4 and in Section 5 we apply it to a mobile phone graph and discuss the results by comparing them to an ethnographic study on communication tools.

\section{PRELIMINARIES}

Let $G=(V, E)$ be a graph; $V$ is the set of its vertices, $E \subseteq V \times V$ is the set of its edges. We define its size as $|V|$, the number of its vertices. Two vertices $u, v \in V$ are adjacent in $G$ if $(u, v) \in E$. The graph $G$ is undirected if, for all $u, v \in V$, there is no difference between $(u, v)$ and $(v, u)$, it is connected if there exists a finite path between every two vertices and it is simple if there is no multiple edge and no self-loop $((v, v) \notin E$, for all $v \in V)$. For a vertex $v \in V$, we denote by $N(v)=\{u \in V,(u, v) \in E\}$ its neighborhood, by $N[v]=N(v) \cup\{v\}$ its closed neighborhood and by $d(v)=|N(v)|$ its degree. The betweenness centrality [13] of a vertex $v$ is defined as $c(v)=\sum_{s, t \in V_{G}} \frac{\delta_{v}(s, t)}{\delta(s, t)}$ where $\delta(s, t)$ denotes the number of shortest paths from $s$ to $t$ and $\delta_{v}(s, t)$ denotes the number of shortest paths from $s$ to $t$ that pass through $v$.

Two graphs $G=\left(V_{G}, E_{G}\right)$ and $H=\left(V_{H}, E_{H}\right)$ are isomorphic if and only if there exists a bijective function $\varphi: V_{G} \rightarrow$ $V_{H}$ (called isomorphism of $G$ and $H$ ) such that any two vertices $u$ and $v$ are adjacent in $G$ if and only if $\varphi(u)$ and $\varphi(v)$ are adjacent in $H$. When $G$ and $H$ are one and the same graph, the function $\varphi$ is called automorphism of $G$. The graph isomorphism is an equivalence relation on graphs so it partitions the class of graphs into equivalence classes, called isomorphism classes.

Given a graph $G=\left(V_{G}, E_{G}\right)$, a graph $H=\left(V_{H}, E_{H}\right)$ is a subgraph of $G$ if $V_{H} \subseteq V_{G}$ and for all $u, v \in V_{H}$, if $(u, v) \in E_{H}$ then $(u, v) \in E_{G} . H$ is an induced subgraph of $G$ (denoted by $H \hookrightarrow G$ ) if $V_{H} \subseteq V_{G}$ and for all $u, v \in V_{H}$, $(u, v) \in E_{H}$ if and only if $(u, v) \in E_{G}$.

For a graph $G$ and an positive integer $k$, Wernicke [36] proposed an algorithm that efficiently enumerates all the connected induced subgraphs of $G$ with exactly $k$ vertices. This algorithm, called $\operatorname{ESU}(G, k)$, starts with a vertex of $G$ and adds neighboring vertices until a set of $k$ vertices is obtained, hence a connected induced subgraph with $k$ vertices. The neighboring vertices must satisfy certain conditions in order to be added to the already selected ones. This guarantees that each subgraph is listed exactly once.

\section{A CHARACTERIZATION OF GRAPHS}

This section introduces a method to characterize a graph and its vertices. Given a graph, we enumerate all its connected induced subgraphs with size at most 5 and we group them into isomorphism classes. Then, for each vertex of the graph, we compute the position it occupies in each one of the found subgraphs. For example, for the graph in Figure $1 a$, the number of its different induced subgraphs and the positions occupied by the vertices $u$ and $v$ are presented in Figure 1 $b, c$ and $d$ respectively.

\subsection{Definitions}

Given a graph $G$ and a vertex $v$ of $G$, we call neighb-degree of $v$, denoted by $n d(v)=\sum_{u \in N[v]} d(u)$, the sum between its degree and the degrees of its neighbors. We call degrees combination of the graph $G$ the ascending sorted list of the neighb-degrees of its vertices. Note that for a graph $G$ with $n$ vertices and $m$ edges one compute the neighb-degrees of all the vertices of $G$ in $O(m)$ time, then its degrees combination in $O(n \cdot \log n)$ time.

Given two graphs $G$ and $H$ and two vertices $u \in V_{G}$ and $v \in$ $V_{H}$, we say that $u$ and $v$ are position equivalent if there exists 


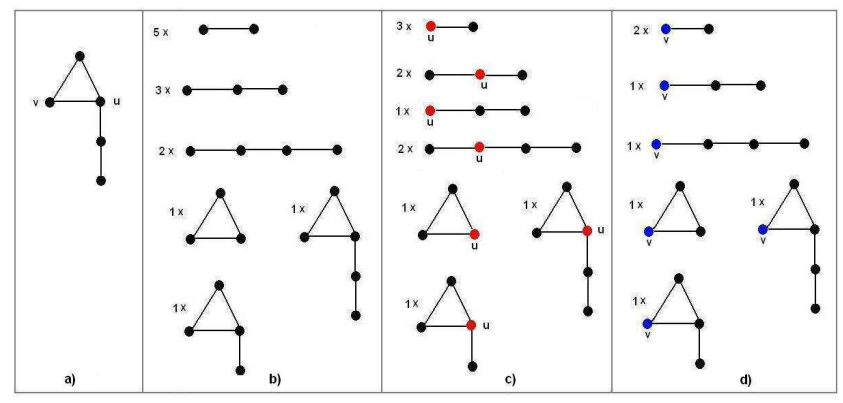

Figure 1: A graph (a), its connected induced subgraphs $(b)$ and the positions of the vertices $u(c)$ and $v(d)$.

an isomorphism $\varphi$ of $G$ and $H$ such that $\varphi(u)=v$. When $G$ and $H$ are one and the same graph, the position equivalence is the automorphic equivalence. In the isomorphism class $C$ of a graph $G$, the set of all vertices is partitioned by the position equivalence into equivalence classes, called the position classes in $C$; we denote by $P(C)$ this set.

We denote by $\mathcal{G}$ the class of all undirected simple graphs, by $\mathcal{C}$ its isomorphism classes and by $\mathcal{P}=\cup_{C \in \mathcal{C}} P(C)$ the set of all the positions; $\mathcal{G}_{k}, \mathcal{C}_{k}$ and $\mathcal{P}_{k}$ are the restrictions of these sets to connected graphs with at most $k$ vertices and at least 1 edge. We call the 30 non-isomorphic graphs with size at most 5 (i.e. the graphs in $C_{5}$ ) patterns. In Figure 2 is represented the set of patterns such that, for each pattern, each color corresponds to a different position class; there are 73 position classes in $\mathcal{C}_{5}$. We sort in ascending order the position classes of a same pattern by their betweenness centrality, then by their degree. We call peripheral the first position class in this order and central the last one. The position classes that are not central nor peripheral or are both central and peripheral are called intermediate.

We define the function $S u b: \mathcal{G} \times \mathcal{C} \rightarrow \mathbb{N}$ such that, for a graph $G$ and an isomorphism class $C, \operatorname{Sub}(G, C)$ counts the number of induced subgraphs of $G$ that belong to $C$ :

$$
\operatorname{Sub}(G, C)=\mid\{H \text { st } H \hookrightarrow G \text { and } H \in C\} \mid .
$$

We define the function Pos $: \mathcal{G} \times \mathcal{P} \times \cup_{G \in \mathcal{G}} V_{G} \rightarrow \mathbb{N}$ such that for a graph $G$, a position class $P$ and a vertex $v \in$ $V_{G}, \operatorname{Pos}(G, P, v)$ counts the number of subgraphs of $G$ that contain $v$ in the position $P$ :

$\operatorname{Pos}(G, P, v)=\mid\left\{H\right.$ st $H \hookrightarrow G, v \in V_{H}, P$ is a position class in the isomorphism class of $H$ and $v \in P\} \mid$.

$S u b_{k}$ and $\operatorname{Pos}_{k}$ are the restrictions of these functions to the sets $\mathcal{C}_{k}$ and $\mathcal{P}_{k}$ respectively.

A new definition of social roles. We introduce here a new definition of equivalence of vertices in order to identify actors that play the same social role. We consider that vertices with the same role must have the same positions with regard to the vertices situated at a certain maximal distance from them. In other words, they must connect in the same way to the vertices around them.

Given a graph $G$ with $n$ vertices and a vertex $v \in V_{G}$, we call position vector of $v$ in $G$ the vector $P s(v)$ such that $\operatorname{Ps}(v)[P]=\operatorname{Pos}(G, P, v)$, for each position class $P \in \mathcal{P}$. The $k$-position vector of $v$, denoted by $P s_{k}(v)$, is the vector computed only for the position classes in $\mathcal{P}_{k}$. Given two vertices $u, v \in V_{G}$ and a positive integer $k \leqslant n$, we call $u$ and $v k$-position equivalent if and only if their $k$-position vectors are identical. In other words, two vertices of $G$ are $k$-position equivalent if and only if, in the induced subgraphs of size at most $k$ of $G$, they occupy the same position the same number of times.

Let us observe that two vertices are 2 -position equivalent if and only if they have the same degree and that two $k$-position equivalent vertices are also $(k-1)$-position equivalent because $P_{k-1} \subset P_{k}$. The $k$-position equivalence is a weaker condition than the structural equivalence: two vertices that are structural equivalent are also $n$-position equivalent; the vice-versa is not always true. It is also weaker than the automorphic equivalence, two vertices being automorphic equivalent if and only if they are $n$-position equivalent.

\subsection{The algorithm}

Given two connected graphs with size at most 5, one can check if they are isomorphic and if two vertices are position equivalent by using only the neighb-degrees of their vertices. This is shown by the following lemma.

Lemma 1. Two graphs $G, H \in \mathcal{G}_{5}$ are isomorphic if and only if their degrees combination are identical. Moreover, if $G$ and $H$ belong to the same isomorphism class $C$, then two vertices $u \in V_{G}$ and $v \in V_{H}$ are position equivalent if and only if they have the same neighb-degree.

Proof. The proof is straightforward, it suffices to check the two statements for all the graphs in $\mathcal{G}_{k}$.

COROLlary 1. Given a graph $G \in \mathcal{G}_{5}$ with $n$ vertices and $m$ edges, its isomorphism class is computed in time $O(m+$ $n \cdot \operatorname{logn})$ and the position classes of its vertices in time $O(m)$.

We propose in Algorithm 1 a method to characterize an undirected simple graph $G$. First we compute the number of occurrences of the 30 patterns as induced subgraphs of $G$ (the array $S b$ ). Then we compute, for each vertex of $G$, its number of occurrences in the position classes of the different patterns (the array $P s$ ).

The choice of limiting the size of the researched induced subgraphs at 5 is motivated by several reasons: the number of isomorphism classes and position classes grows with the size, so the time and memory complexities increase. Moreover, when limiting the size at 5 , we dispose of a very efficient method to compute the different equivalence classes, hence the number of their occurrences.

For the first part of the characterization method (line 1), we use the algorithm $\operatorname{ESU}(G, k)[36]$ with $k \leqslant 5$. For the second task (line 2), we apply Lemma 1 , so we compute the neighb-degrees of the vertices in each subgraph. The time complexity of this algorithm is linear in the number of 


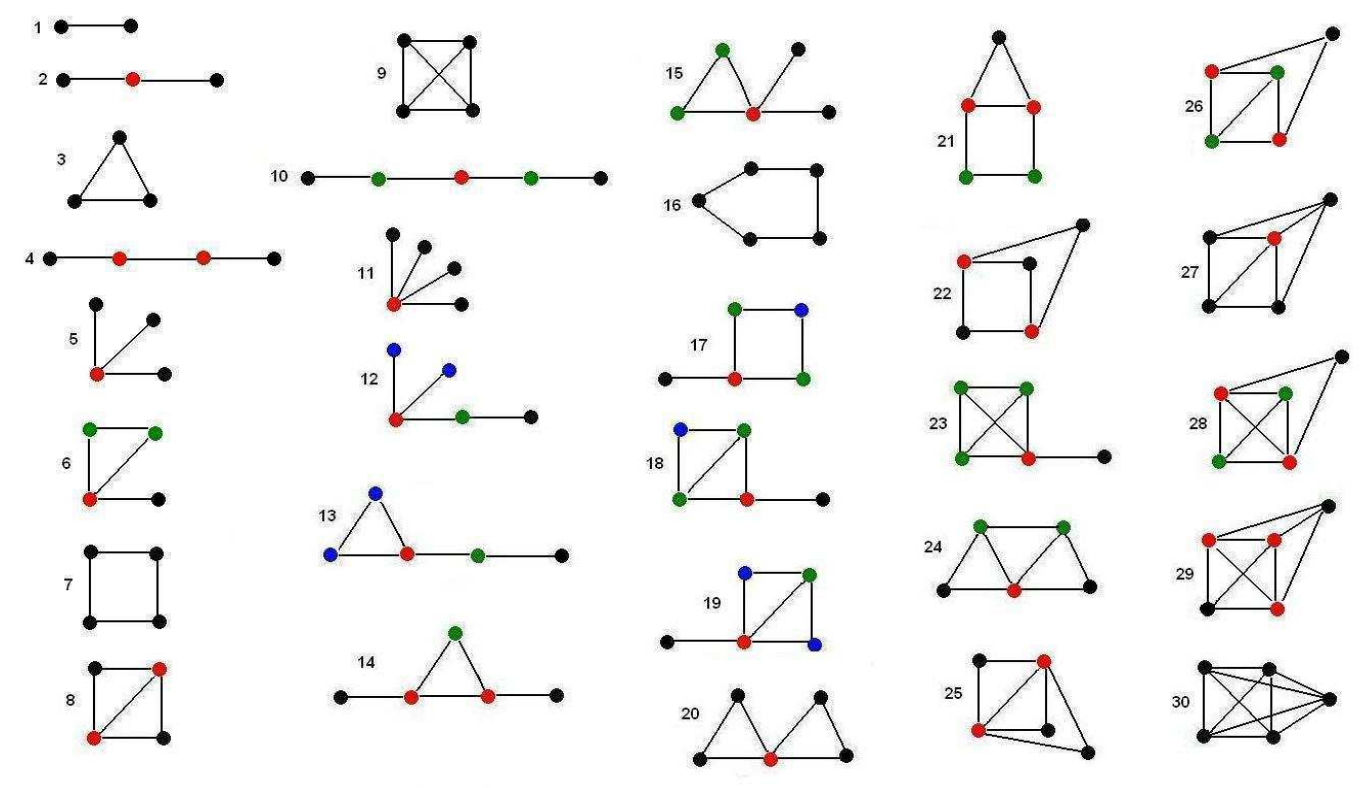

Figure 2: The sets of patterns and their positions. The order of the colors is black (1), blue (2), green (3) and red(4) corresponding to the ascending order of centrality and degree.

\author{
Algorithm 1 characterize. Characterizes an undirected \\ simple graph \\ Input: A set of edges representing an undirected simple graph $G$ \\ Output: An array $S b$ such that $S b[C]=S u b_{5}(G, C)$ and \\ an array $P s$ such that $P s[v][P]=\operatorname{Pos}_{5}(G, P, v)$ \\ 1. enumerate all the connected induced subgraphs of $G$ \\ of size at most $5 \Rightarrow$ the set $S$ \\ 2. for each graph $H \in S$ \\ 2.1. find its isomorphism class $C$ and increment $S b[C]$ \\ 2.2. for each vertex $v$ of $H$, find its position class $P$ in $C$ \\ and increment $P s[v][P]$
}

connected induced subgraphs of size at most 5 of the input graph: for the first part (the enumeration), see [36]; for the second part, note that it takes a constant time to compute the isomorphism class and the position classes of each subgraph found in the first part (see Corollary 1). As for the space complexity, note that one doesn't need to explicitly build and store the set $S$, but only one subgraph at a time. When a connected induced subgraph of $G$ of size at most 5 is found, its isomorphism class and the position classes of its vertices are computed before proceeding to the search of another subgraph.

Note that Algorithm 1 can be easily modified in order to enumerate all the connected induced subgraphs with a maximal number of edges. As in the case of the graphs with at most 5 vertices, the isomorphism class of a graph with at most 6 edges can be identified using only the degrees combination. Moreover, two vertices $u$ and $v$ belonging to two isomorphic graphs with at most 6 edges are position equivalent if and only if

$$
\sum_{t \in N[u]} n d(t)=\sum_{t \in N[v]} n d(t)
$$

\section{NEIGHBORHOODS}

In this section, we propose a method (Algorithm characterize_neighborhoods) to analyze the local structure of a large graph using the notions introduced in the previous section. For each vertex $v$ of a given large graph $G$, we first compute the subgraph $G n(v)$ induced by the neighbors of $v$, so we need to list the triangles containing $v$. The latter problem has been extensively studied in [23]; we rely on Algorithm new-vertex-listing proposed in this paper in order to compute, for each vertex $v \in G M$, the subgraph $G n(v)$.

We characterize the obtained neighborhood graph $G n(v)$ using Algorithm 1, so we compute the number of occurrences of each pattern in $G n(v)$ (the array $S b$ in Algorithm 1) and the position classes occupied by the neighbors of $v$ (the array $P s$ in Algorithm 1). Using the arrays $S b$ and $P s$ we update two global arrays $S^{\prime}$ and $P^{\prime}$. The first one contains, for each pattern, its total number of occurrences in the neighborhood graphs of the given large graph. After having associated, using extra-data, different types to the neighbors of each vertex in the large graph, one can compute the second array: the number of occurrences in each position class of the vertices of a certain type. We detail the updating of the two arrays in the next section.

As in Algorithm new-vertex-listing of [23], we use the adjacency matrix of $G$ (the array $A$ ) without explicitly storing it, this way being able to test for any edge $(v, w)$ in $O(1)$ time and space. The array $A$ is built in $O\left(\left|V_{G}\right|\right)$ time and space at the beginning of the algorithm and is then just modified in time $O(d(v))$ for each vertex $v$. Thus, for each vertex $v$, the subgraph $G n(v)$ induced by its neighbors is computed 
$\overline{\text { Algorithm } 2 \text { characterize_neighborhoods. Characterizes }}$ the neighborhood of each vertex of a large graph

Input: An undirected simple large graph $G$

Output: Two arrays $S^{\prime}$ and $P^{\prime}$

1. create an array $A$ of $\left|V_{G}\right|$ integers and set them to -1

2. for each vertex $v$ of the graph $G$

2.1. initialize $E$ to the empty set

2.2. for each vertex $u$ in $N(v)$, set $A[u]$ to $v$

2.3. for each vertex $u$ in $N(v)$

2.3.1. for each vertex $w$ in $N(u)$ if $A[w]=v$ then add $(w, u)$ to $E$

2.4. characterize $(E)$

2.5. update $S^{\prime}$ and $P^{\prime}$

in time $O\left(\sum_{u \in N[v]} d(u)\right)$. Now, the complexity $O(d(u))$ will be added for each one of the neighbors of $u$ which give a time complexity $O\left(\sum_{v \in G} d^{2}(v)+\left|V_{G}\right|+\left|E_{G}\right|\right)$ for the entire set of vertices of the large graph $G$.

In the next section, we apply Algorithm 2 to a large social graph. We also present the degree and triangle distributions, important parameters for the complexity of our method.

\section{ANALYSIS OF A LARGE SOCIAL GRAPH \\ 5.1 Description of the graph}

We analyze a large graph built from a mobile phone database. The database contains a month of mobile phone communications (phone calls and short messages) between the clients of a same operator in a European country. We build a graph where the vertices are the clients; we connect such two vertices by an undirected edge if each of the two persons has contacted at least once the other person during the recorded month. This way we don't take into consideration the oneway contacts (calls or messages), single events in most of the cases suggesting that the two individuals don't know each other personally. We obtain a graph (that we call $G M$ ) with $2.7 \times 10^{6}$ vertices and $6.4 \times 10^{6}$ edges; $83 \%$ of its vertices and $99 \%$ of its edges belong to the same giant connected component.

For each vertex (ego) $v$ in $G M$, we study the graph $G n(v)$ induced by its neighborhood, so we analyze $2.7 \times 10^{6}$ graphs; we denote by $D=\{G n(v), v \in G M\}$ this set of neighborhood graphs. As expected, most of these graphs have a small number of vertices (this number is equal to the degree of $v$ ) while only a small minority have a great number of vertices. The same statement is valid for the number of edges of each graph in $D$ (this number is equal to the number of triangles containing ego). Table 1 contains the minimum, maximum, median and average values of the two parameters, as well as the number of graphs in $D$ where the value of the parameter is greater than 100. Figure 3 contains the distribution of the number of vertices and of the number of edges of the graphs in $D$. Only 20 graphs (i.e. $7 \times 10^{-4} \%$ ) have more that 100 vertices and more that 100 edges. The average of the densities of the graphs in $D$ is the clustering coefficient of $G M$, equal to 0.097 .

Empirical complexity of the method. Let us discuss

\begin{tabular}{|l|l|l|l|l|l|}
\hline $\begin{array}{l}\text { parameter } \\
\alpha\end{array}$ & min & max & average & median & $\begin{array}{l}\text { nb. net- } \\
\text { works s. t. } \\
\alpha>100\end{array}$ \\
\hline$n$ & 0 & 367 & 4.66 & 3 & 56 \\
\hline$m$ & 0 & 887 & 2.28 & 1 & 560 \\
\hline
\end{tabular}

Table 1: Different measures for the number of vertices $(n)$ and the number of edges $(\mathrm{m})$ of the $2.7 \times 10^{6}$ neighborhood networks

the complexity of the method characterize when it is applied to the graphs in $D$, i.e. when Algorithm 2 is applied to the mobile phone graph $G M$. For a graph $G$ in $D$, let $n_{G}$ be its number of vertices, $m_{G}$ its number of edges and $s_{G}$ its number of patterns. As we explained in the previous section, the time complexity of characterize $(G)$ is $O\left(s_{G}\right)$. After applying Algorithm 2 to $G M$, we know, for each graph $G \in D$, the number $s_{G}$, so we are able to empirically determine the complexity of Algorithm 2 on $G M$. For all graphs $G \in D$, we have $s_{G}<m_{G}^{3}$ and for $98.5 \%$ of these graphs $s_{G}<m_{G}^{2}$, so, on our graph $G M$, the observed time complexity of the method characterize $(G)$ is $O\left(m_{G}^{2}\right)$ in $98.5 \%$ of the cases and $O\left(m_{G}^{3}\right)$ in the rest of the cases. Given that the graphs in $D$ are not very dense, it is not very time-consuming to list all the induced subgraphs instead of just counting them. We compared the time complexity of the method proposed by Kloks et al. [20] that counts the induced subgraphs with exactly 4 vertices to that of the method proposed by Wernicke [36], that we use in Algorithm 1. On the one hand, for a graph $G$ with $n_{G}$ vertices, the complexity of Kloks' algorithm is $O\left(n_{G}^{\alpha}+e^{1.69}\right)$, where $O\left(n_{G}^{\alpha}\right)$ is the time needed to compute the square of the adjacency matrix of $G$. On the other hand, for each graph $G \in D$, the number of induced subgraphs with 4 vertices is smaller than $\left(2 \times m_{G}\right)^{2}$ and than $\left(5 \times n_{G}\right)^{2}$. Therefore, for the mobile phone graph $G M$, the time complexities of the two methods are comparable. Then it is worth listing all the subgraphs, given that we make a step further by computing not just the number of the different subgraphs but also the position classes of the vertices.

\subsection{Characteristic patterns}

We address here the problem of identifying the patterns that are "characteristic" for the set $D$ of neighborhood graphs. There are several possible definitions for a characteristic pattern $C$ for a set of graphs $D$ :

Def 1 . the number of occurrences of the pattern $C$ as induced subgraph of the graphs in $D$ is greater than a given threshold;

Def 2. the number of graphs in $D$ that contain the pattern $C$ as induced subgraph is greater than a given threshold (this is the adopted approach in $[17,19,21,39]$ );

Def 3. the number of occurrences of the pattern $C$ as induced subgraph is higher for the graphs in $D$ than for randomly generated graphs of same sizes (this approach was introduced in [28]).

Def 1 . We computed, for each pattern $C$ with $k \leqslant 5$ vertices, the number of occurrences of $C$ as induced subgraph 


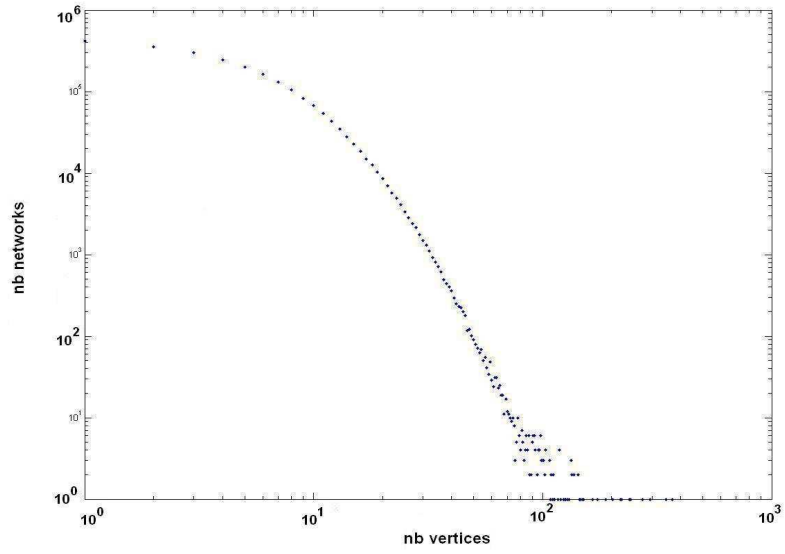

Figure 3: The distribution of the number of vertices neighborhood networks

in $D$ divided by the number of occurrences of a pattern with $k$ vertices in $D$, i.e. the probability that the subgraph induced by $k$ connected vertices of a graph in $D$ represents the pattern $C$. Figure 4 (left) contains the values of these probability for $k>3$. We observe that the patterns that occur the most are the paths and the stars (possibly with an extra edge). Of course the counting of all the occurrences of a certain pattern gives an advantage to those containing vertices of degree 1 .

Def 2. Figure 4 (right) contains, for each pattern $C$ with $k \leqslant 5$ vertices, the number of graphs in $D$ that contain $C$ as induced subgraph divided by the number of graphs in $D$ that contain at least one pattern with $k$ vertices, i.e. the probability that a graph in $D$ with at least $k$ connected vertices contains $C$. We observe that the most frequent patterns are the paths, possibly with one extra edge (added to form a star or a triangle).

Def 3. For each connected component of a graph in $D$ we randomly generated connected graphs using the method introduced in [27]. This method computes $d K$-series of probability distributions (i.e. all degree correlations within $d$-sized subgraphs).We built graphs for $d=1,2$ and 3 respectively. For $d=1$, the generated graphs preserve the degree distribution of the original graphs, thus assuring also the same number of vertices and edges. For $d=2$, the joint degree distribution is preserved, thus keeping also the same degree distribution. For $d=3$, the graph generation preserves the number of triangles and wedges (i.e. chains of 3 vertices connected by 2 edges) between vertices with degrees $k_{1}, k_{2}, k_{3}, \forall k_{1}, k_{2}, k_{3} \in \mathbb{N}$.

For each value of $d$, let $R_{d}$ be the set of randomly generated graphs. Note that all the three generations (for $d=$ $1,2,3)$ preserve the degree and the clustering coefficient of the graph GM. For each pattern, we computed the ratio between its number of occurrences in the graphs in $D$ and in the graphs in $R_{d}$. When the graphs in $D$ are compared to the graphs in $R_{d}$, the patterns with the greatest values of the ratios are characteristic for the the graphs in $D$ and the ones with the smallest values are characteristic for the

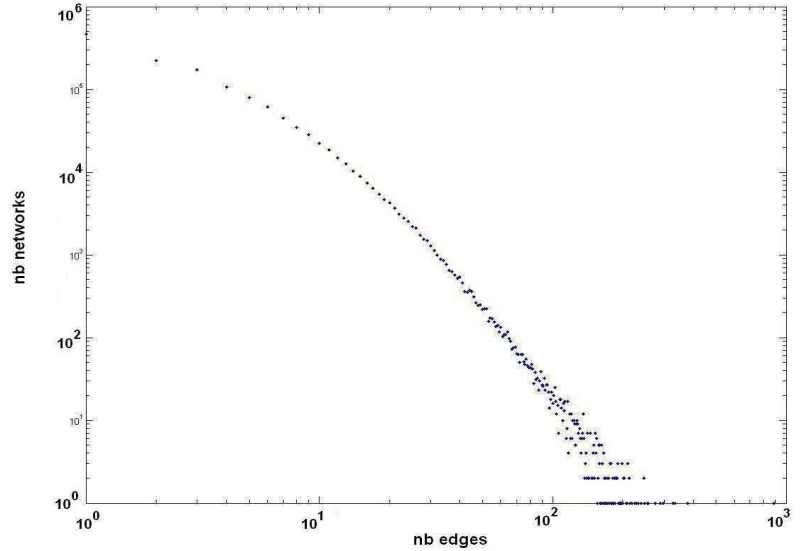

(a) and of the number of edges $(b)$ of the $2.7 \times 10^{6}$ graphs in $R_{d}$. For $d=1$ and $d=2$, the same patterns are identified as characteristic (see Figure 5), with smaller values of the ratio for $d=2$ than for $d=1$. These patterns suggest that, although the densities of the input graphs are preserved in the generated ones, there are graphs in $D$ that are locally more dense than the corresponding generated ones. So, in the neighborhood of certain vertices, several neighbors form dense clusters even though they belong to the same connected component; these clusters may correspond to the different groups of contacts of that person. Note however that the two generations don't preserve the clustering coefficients of the graphs in $D$. When $k=3$, the clustering coefficient is preserved and the observed values of the ratio are placed between 0.99 and 1.003 for all the patterns. The generated graphs essentially reconstruct the original ones, so the $3 k$-distribution suffices in order to capture the distributions of the different patterns in the neighborhood graphs in $G M$. Nevertheless, this generation is very constraining for small graphs like those in $D$; in many cases there is only one graph that has the $3 k$-distribution of the original one: the original one. Besides, the structure of neighborhoods refers not only to the distributions of the different patterns, but also to the roles played by the vertices; this question is discussed in the next section.

\subsection{Positions of the vertices}

Recall that by applying Algorithm 2 to the graph $G M$ we computed, for each ego $v$ in $G M$ and each vertex $u$ in the neighborhood $G n(v)$ of $v$, the positions of $u$ in $G n(v)$. We analyze here, for each ego $v$ and each vertex $u$ in $G n(v)$, the relation between the positions occupied by $u$ in $G n(v)$ and the quantity of its communications with $v$. Note that the positions of $u$ are completely determined by its links with the other vertices in $G n(v)$; the quantity of communication of $u$ with ego $v$ will also be relativized to the quantity of communication between ego and the other persons in his network.

\subsubsection{The maximal number of calls}

First, for each ego $v$, we index his neighbors depending on the number of calls they exchanged with him: the greater the number of calls exchanged with ego, the smaller the index, 

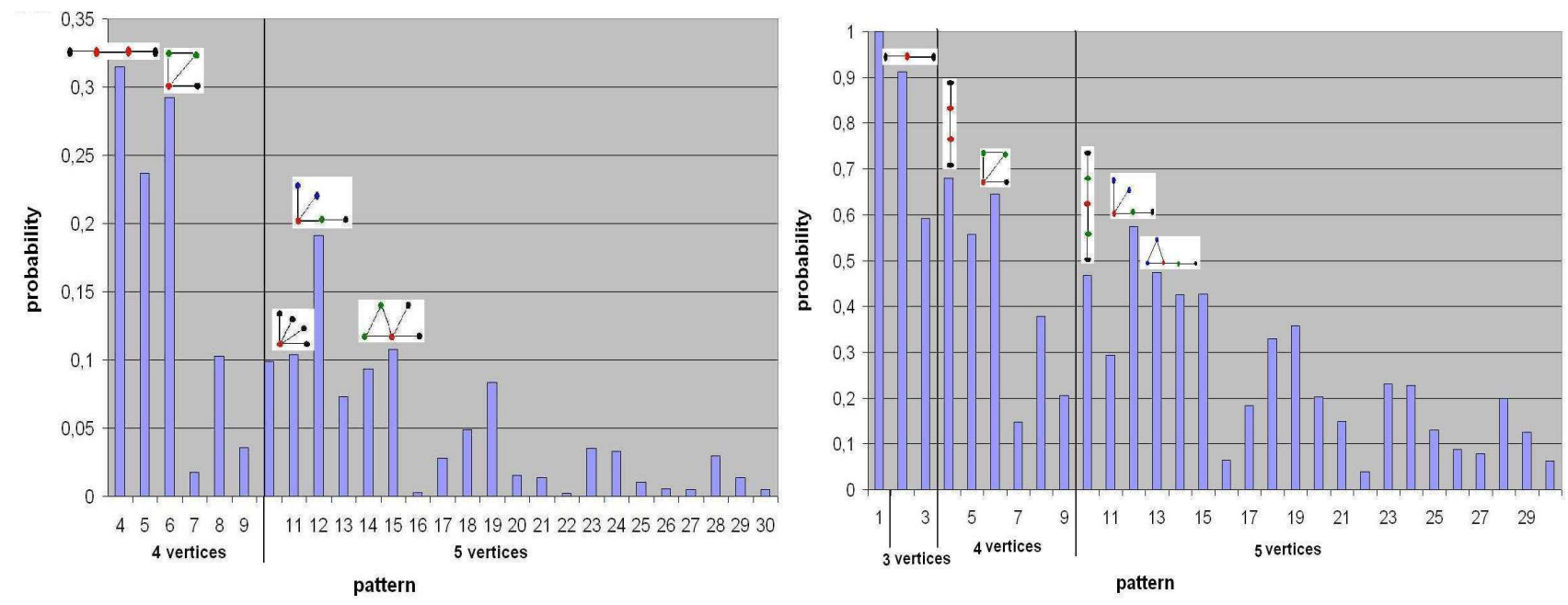

Figure 4: For each pattern with $k$ vertices, the probability: to be the subgraph induced by $k$ connected vertices in $D$ (left) and to occur in a graph in $D$ that has at least $k$ connected vertices (right)

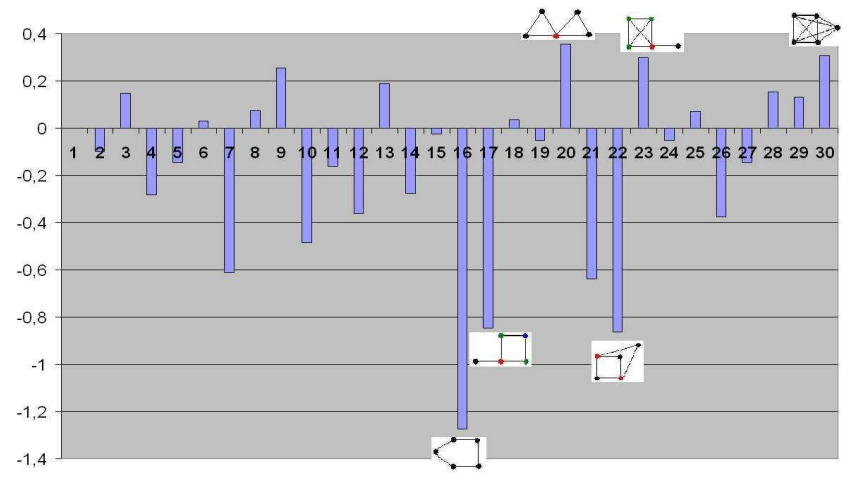

Figure 5: For each pattern, $\log _{2}$ of the ratio between its $\mathrm{nb}$ of occurrences in $D$ and in $R_{2}$

such that the vertex with the greatest number of calls has index 1 and the one with the smallest has index $d(v)$.

Let $D_{5}$ be the set of graphs in $D$ with at least 5 vertices, i.e. the set of neighborhood graphs of the vertices in $G M$ with degree at least 5 . For each graph in $D_{5}$, we study the positions occupied by its vertices with indices $1,2,3$ and 4 and by a randomly chosen vertex between those with index greater than 4 to which we give the index 0 . In order to do that, we answer two questions regarding the entire set $D_{5}$ :

Q1 given a position class in $\mathcal{P}_{5}$, which of the five indices occupies this position the most frequently and which one the least frequently?

Q2 given a class $C \in \mathcal{C}_{5}$ and an index $i<5$, in which position class of $C i$ appears the most frequently and in which one the least frequently?

For an index $i<5$, let $I(i)$ be the set of vertices that have index $i$ in the graphs in $D_{5}$ along with the corresponding graphs: $I(i)=\left\{(u, G)\right.$ s.t. $u \in V_{G}, G \in D_{5}$ and $\left.\operatorname{index}(u)=i\right\}$. For an index $i<5$ and a position class $P \in \mathcal{P}_{5}$, we define:

- the absolute frequency of occurrence $F a(i, P)$ of $i$ in $P$ as the total number of occurrences in $P$ of the vertices with index $i$ divided by the sum of degrees of the vertices with index $i$ :

$$
F a(i, P)=\frac{\sum_{(u, G) \in I(i)} \operatorname{Pos}_{5}(G, P, u)}{\sum_{(u, G) \in I(i)} d(u)} .
$$

- the relative frequency of occurrence $\operatorname{Fr}(i, P)$ of $i$ in $P$ as the number of graphs in $D_{5}$ where a vertex with index $i$ appears in the position $P$ divided by the number of graphs in $D_{5}$ where a vertex with index $i$ has the degree at least 1 :

$$
F r(i, P)=\frac{\left|\left\{(u, G) \in I(i), \operatorname{Pos}_{5}(G, P, u)>0\right\}\right|}{|\{(u, G) \in I(i), d(u)>0\}|}
$$

Question $Q 1$. For each position class $P \in \mathcal{P}_{5}$, we sorted respectively the five values $F a(i, P)$ and the five values $F r(i, P)$, for $i \in\{0,1,2,3,4\}$. We find that, for most of the central position classes, the vertices with index 1 occur more often than the ones with index 2 and they occur more often than the ones with index 3 etc; the randomly selected vertices occur the least often in these central position classes. The opposite situation happens for most of the peripheral position classes, that are occupied the most frequently by the randomly chosen vertices and the least frequently by the ones with index 1 . As for the intermediate positions, they are mostly occupied by the vertices with indices 3 and 4 . The position classes of all the 30 isomorphism classes follow this tendency, except for those of the five following classes: 8, 21, 22, 26 and 29. Note however that in these cases the difference between central and peripheral is not very sharp and that these classes are not very frequent (see Figure 4).

Question Q2. For each isomorphism class $C \in C_{5}$ and each index $i \in\{0,1,2,3,4\}$, we sorted the values $F a(i, P)$ of 
the position classes $P$ of $C$. We observe that, for all of the isomorphism classes (except 10 and 27), the vertices with index 1 occupy most frequently the central positions and least frequently the peripheral ones. The randomly chosen vertex occupies mostly the peripheral positions and least frequently the central ones, while the vertices with indices 2, 3 and 4 have a tendency placed between these two.

These results allow us to make the following statement: in most of the induced subgraphs where it appears, the person that exchanged the greatest number of calls with ego plays a central role, being a connection point between several other neighbors. The roles of the next three vertices are less central, but they remain more central than that of the randomly chosen neighbor. Note that this centrality of roles is identified using the small induced subgraphs where the vertices appear. This means that a vertex has a more or less important position with regard to the vertices that are around it and not to the whole graph. This corresponds to our aim of computing the roles of the vertices rather than measure their centrality.

\subsubsection{The maximal sum of duration of calls}

We analyze, for the network of each ego, the position classes occupied by the vertex that had the greatest sum of duration of calls with ego. In $78.2 \%$ of the cases, the person that exchanged the greatest number of calls with ego (the vertices with index 1 of the previous section) is also the person that has the greatest total duration. In the other cases, we gave index 1 to the vertex with the greatest number of calls and index 2 to the vertex with the greatest sum of duration of calls. We also randomly chose a vertex among the other neighbors of ego. We observe that the vertices with index 2 appear less often in a central position that the vertices with index 1 but more often that the randomly chosen vertices. The vertices with index 2 prefer the intermediate positions.

\subsection{Interpretation of the results}

We rely on the results of several studies in order to try to explain the positions occupied by the two types of contacts of ego: the ones with the greatest frequency of calls and the one with the greatest total duration. In [25], Licoppe et Smoreda analyzed the relation between social networks, exchanges between actors and communication tools using databases of telephone calls, Internet traffic and several interviews focusing on the use of telephone. For a pair of actors, they identified two patterns of relationship and the corresponding communication tools. The first pattern is that of "connected presence", where the two persons, socially and often also geographically close, are frequently in contact with each other, exchanging many short calls and messages. They share activities that require numerous calls for synchronization and coordination, the mobile phone being especially suitable for this. In our network, the person that exchanged the greatest number of calls with ego has an important role in the network of neighbors, occupying central positions more often than other vertices and often connecting neighbors otherwise disconnected. The next three persons have also more important positions than a randomly selected neighbor. The second pattern identified by Licoppe et Smoreda is that of "intermittent presence", where the two persons, close friends or intimate relatives, are not able to see each other or talk very often. Their conversations are long, they give and re- ceive news, trying to compensate for the rarity of face-to-face contacts. In our network, the person that has the greatest sum of duration of calls with ego, when he doesn't have the greatest frequency too, occupies more important positions than a randomly selected neighbor. It has however a less central role than the person that has the most frequent calls.

The relation between the geographical distance between two persons and the probability of existence of a link connecting them has also been studied [22, 24]. In [22], Lambiotte et al. showed that in a mobile phone network this probability is inversely proportional to the square of the geographical distance. According to [24], a similar tendency is observed in a different kind of large social networks (bloggers community): the probability of existence of a link is inversely proportional to the number of closer friends. Therefore, one can imagine that in most of the neighborhoods in our mobile phone graph many of ego's contacts are geographically close to him. The person that has long, but rare calls with ego probably doesn't see him very often; it is not surprising that this person's position is not as important as that of the contact with the greatest frequency. The duration of the calls suggests though a certain social closeness between this person and ego; he probably knows and calls some of ego's contacts, which explains his more important position than that of a randomly chosen neighbor. Imagine, for instance, that the persons that have frequent contacts with ego are his friends or colleagues that he sees nearly daily and the person that has the greatest sum of durations is a childhood friend.

\section{CONCLUSIONS AND PERSPECTIVES}

We presented in this paper a method for analyzing the local structure of large graphs that we applied to a $2.7 M$ vertices, $6 M$ edges mobile phone graph. In the neighborhood of each vertex (called ego) of the graph, we listed all the patterns and we identified the characteristic ones. Then we addressed the notion of role in a graph, focusing on the position occupied by each vertex and the importance of this position. Our goal was not to define a certain centrality of the vertices, but rather to find how they connect to each other, how they are placed and how important they are in the local structures they form with the other vertices.

A step further could be to compare neighborhood graphs for instance by merging vertices having "similar" functions. We believe indeed that the identification of patterns and positions of vertices can offer the context for new definitions of similarity. We hope that future research based on the notions introduced in this paper will provide new methods for measuring the similarity between graphs and between roles of vertices. It would also be interesting to apply this method to another kind of large graphs, for instance to online communities where the neighborhoods are more dense but the connections between people are weaker.

\section{REFERENCES}

[1] R. Agrawal and R. Srikant. Fast algorithms for mining association rules in large databases. In $V L D B$ '94.

[2] A.-L. Barabasi and R. Albert. Emergence of scaling in random networks. Science, 1999. 
[3] F. Barth. Political leadership among the Swat Pathans. Athlone Press, London, 1959.

[4] J. Boissevain. Friends of Friends, Networks, Manipulators and Coalitions. Basil Blackwell, Oxford, 1974.

[5] S. Borgatti and M. Everett. The class of all regular equivalences: Algebraic structure and computation. Social Networks, 11(1):65-88, March 1989.

[6] S. Bornholdt and H. G. Schuster, editors. Handbook of Graphs and Networks. Wiley-Vch, 2003.

[7] E. Bott. Family and Social Network. Tavistock, London, 1957.

[8] R. Breiger, S. Boorman, and P. Arabie. An algorithm for clustering relational data with applications to social network analysis and comparison with multidimensional scaling. Journal of Mathematical Psychology, 12:328-383, 1975.

[9] S. Brin and L. Page. The anatomy of a large-scale hypertextual Web search engine. Computer Networks and ISDN Systems, 30(1-7):107-117, 1998.

[10] J. Candia, M. C. González, P. Wang, T. Schoenharl, G. Madey, and A. Barabási. Uncovering individual and collective human dynamics from mobile phone records. Journal of Physics A: Mathematical and Theoretical, 41(6):224015+, June 2008.

[11] K. Efe, V. Raghavan, C. H. Chu, A. L. Broadwater, L. Bolelli, and S. Ertekin. The shape of the Web and its implications for searching the Web, 2000.

[12] G. Flake, S. Lawrence, and C. L. Giles. Efficient identification of web communities. In Sixth $A C M$ SIGKDD International Conference on Knowledge Discovery and Data Mining, pages 150-160, Boston, MA, 2000

[13] L. Freeman. A set of measures of centrality based on betweenness. Sociometry, 40:35-41, 1977.

[14] D. Gibson, J. M. Kleinberg, and P. Raghavan. Inferring web communities from link topology. In ACM Conference on Hypertext and Hypermedia, pages 225-234, 1998.

[15] J. Han, J. Pei, and Y. Yin. Mining frequent patterns without candidate generation. SIGMOD Rec., $29(2): 1-12,2000$

[16] C. Haythornthwaite. Social networks and internet connectivity effects. Information, Communication and Society, 8(2):125-147, June 2005.

[17] H. He and A. Singh. Graphrank: Statistical modeling and mining of significant subgraphs in the feature space. In ICDM '06.

[18] B. Hogan. The Handbook of Online Research Methods, chapter Analyzing Social Networks via the Internet. Sage: Thousand Oaks, CA, 2008.

[19] A. Inokuchi, T. Washio, and H. Motoda. An apriori-based algorithm for mining frequent substructures from graph data. In PKDD '00.

[20] T. Kloks, D. Kratsch, and H. Müller. Finding and counting small induced subgraphs efficiently. Information Processing Letters, 74(3-4):115-121, 2000.

[21] M. Kuramochi and G. Karypis. Frequent subgraph discovery. In $I C D M$ ' 01.

[22] R. Lambiotte, V. Blondel, C. de Kerchove, E. Huens, C. Prieur, Z. Smoreda, and P. V. Dooren.
Geographical dispersal of mobile communication networks. Physica A, 387(21):5317-5325, September 2008.

[23] M. Latapy. Main-memory triangle computations for very large (sparse (power-law)) graphs. Theoretical Computer Science (TCS), (407):458-473, 2008.

[24] D. Liben-Nowell, J. Novak, R. Kumar, P. Raghavan, and A. Tomkins. Geographic routing in social networks. Proceedings of the National Academy of Sciences, 102(33):11623-11628, August 2005.

[25] C. Licoppe and Z. Smoreda. Are social networks technologically embedded? how networks are changing today with changes in communication technology. Social Networks, 27(4):317-335, October 2005.

[26] F. Lorrain and H. White. Structural equivalence of individuals in social networks. Journal of Mathematical Sociology, 1:49-80, 1971.

[27] P. Mahadevan, D. Krioukov, K. Fall, and A. Vahdat. Systematic topology analysis and generation using degree correlations. SIGCOMM Comput. Commun. Rev., 36(4):135-146, 2006.

[28] R. Milo, S. Shen-Orr, S. Itzkovitz, N. Kashtan, D. Chklovskii, and U. Alon. Network motifs: Simple building blocks of complex networks. Science, 298(5594):824-827, October 2002.

[29] M. E. J. Newman. The structure and function of complex networks. SIAM Review, 167(45), 2003.

[30] J. P. Onnela, J. Saramäki, J. Hyvönen, G. Szabó, D. Lazer, K. Kaski, J. Kertész, and A. L. Barabási. Structure and tie strengths in mobile communication networks. Proceedings of the National Academy of Sciences, 104(18):7332-7336, May 2007.

[31] C. Prieur, A. Stoica, and Z. Smoreda. Extraction de réseaux égocentrés dans un (très grand) réseau social. Bull. de Méthodologie Sociol., (101), 2009.

[32] J. Scott. Social Network Analysis. Sage, London, 1992.

[33] S. Wasserman and K. Faust. Social Network Analysis: Methods and Applications. Cambridge University Press, 1994.

[34] D. Watts and S. Strogatz. Collective dynamics of small-world networks. Nature, 393, 1998.

[35] B. Wellman. An egocentric network tale. Social Networks, 15:423-436, 1993.

[36] S. Wernicke. Efficient detection of network motifs. IEEE/ACM Trans. Comput. Biol. Bioinformatics, 3(4):347-359, 2006.

[37] H. White, S. Boorman, and R. Breiger. Social structure for multiple networks i. blockmodels of roles and positions. American Journal of Sociology, 81, 1976.

[38] X. Yan, H. Cheng, J. Han, and P. Yu. Mining significant graph patterns by leap search. In SIGMOD '08.

[39] X. Yan and J. Han. gspan: Graph-based substructure pattern mining. In ICDM '02. 